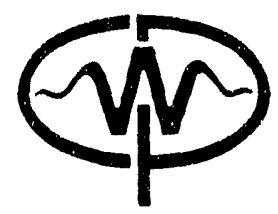

\title{
Dip-Moveout Error in Transversely Isotropic Media with Linear Velocity in Depth
}

\author{
Ken Larner
}

\section{DISCLAIMER}

\begin{abstract}
This report was prepared as an account of work sponsored by an agency of the United States Government. Neither the United States Government nor any agency thereof, nor any of their employees, makes any warranty, express or implied, or assumes any legal liability or responsibility for the accuracy, completeness, or usefulness of any information, apparatus, product, or process disclosed, or represents that its use would not infringe privately owned rights. Reference herein to any specific commercial product, process, or service by trade name, trademark, manufacturer, or otherwise does not necessarily constitute or impiy its endorsement, recommendation, or favoring by the United States Government or any agency thereof. The views and opinions of authors expressed herein do not necessarily state or reflect those of the United States Government or any agency thereof.
\end{abstract}

Center for Wave Phenomena

Colorado School of Mines

Golden, Colorado 80401

303/273-3557 


\title{
Dip-moveout error in transversely isotropic media with linear velocity variation in depth
}

\author{
Ken Larner
}

\begin{abstract}
Levin (1990) modeled the moveout, within CMP gathers, of reflections from plane-dipping reflectors beneath homogeneous, transversely isotropic media. For some media, when the axis of symmetry for the anisotropy was vertical, he found departures in stacking velocity from predictions based upon the familiar cosine-ofdip correction for isotropic media. Here, I do similar tests, again with transversely isotropic models with vertical axis of symmetry, but now allowing the medium velocity to vary linearly with depth.

Results for the same four anisotropic media studied by Levin show behavior of dip-corrected stacking velocity with reflector dip that, for all velocity gradients considered, differs little from that for the counterpart homogeneous media. As with isotropic media, traveltimes in an inhomogeneous, transversely isotropic medium can be modeled adequately with a homogeneous model with vertical velocity equal to the vertical rms velocity of the inhomogeneous medium.

In practice, dip-moveout (DMO) is based on the assumption that either the medium is homogeneous or its velocity varies with depth, but in both cases isotropy is assumed. It turns out that for only one of the transversely isotropic media considered here-shale-limestone-would $v(z)$ DMO fail to give an adequate correction within CMP gathers. For the shale-limestone, fortuitously the constant-velocity DMO gives a better moveout correction than does the $v(z)$ DMO.
\end{abstract}

\section{INTRODUCTION}

Given the complexity possible for subsurface structure, moveout for reflections within common-midpoint (CMP) gathers can have all manner of complication. Nevertheless, the approximation of that moveout for $\mathrm{p}$-wave reflections is hyperbolic over 
typical spreadlengths is adequate in much routine practice. Moreover, Levin (1979) and (1990) has shown that the hyperbolic approximation remains valid for homogeneous, transversely isotropic media. (A transversely isotropic medium is one in which the velocity of plane waves - the so-called phase velocity-varies with direction of propagation from an axis of symmetry, but is independent of direction within the plane perpendicular to the symmetry axis.) Levin (1979) considered reflections from horizontal interfaces in homogeneous, transversely isotropic media with vertical axis of symmetry. He showed that the stacking velocity, both for finite-length offset and in the limit of small source-to-receiver offset, could depart substantially from the propagation velocity in the vertical direction.

For each wave type-sh, (quasi)sv, or (quasi)p-the departure is a complicated function of the five elastic moduli that characterize a transversely isotropic medium. For sh-waves, for exarnple, the stacking velocity exactly equals the velocity of propagation in the horizontal direction. For p-waves, depending on the ratios among the four pertinent elastic moduli, the stacking velocity may approximate either the vertical or horizontal velocity; it may be somewhere between the two; or it can actually be larger or smaller than both (compare columns 6 and 7 of Table 1).

In his theoretical analysis of properties of weakly anisotropic media, Thomsen (1986) obtained an expression for small-offset stacking velocity for $\mathrm{p}$-wave reflections from a horizontal interface. There, he showed that both the size and magnitude of the departure of stacking velocity from vertical velocity are governed by a measure of anisotropy that is totally unrelated to the ratio of horizontal to vertical velocity-the most commonly quoted measure of p-wave anisotropy.

In numerical studies of traveltimes of reflections from plane-dipping interfaces beneath homogeneous, transversely isotropic media, Levin (1990) found that moveout remains approximately hyperbolic and that stacking velocity depends on the orientation of the symmetry axis for the anisotropy, as well as on reflector dip. When the symmetry axis is normal to the reflecting plane, he found that the stacking velocity $V_{\text {stack }}(\phi)$ for reflector dip $\phi$ satisfies

$$
V_{\text {stack }}(\phi) \approx V_{\text {stack }}(0) / \cos \phi .
$$

Thus, for such symmetry, stacking velocity can be "dip-corrected" with the same $\cos \phi$ correction that is used for reflections from a dipping interface beneath a homogeneous, isotropic medium.

For all of the media he studied (see Table 1), Levin found more complicated behavior of stacking velocity with reflector dip when the axis of symmetry is vertical. His shale-limestone medium exhibited the most extreme behavior. For that medium, for example, $\cos \phi$-corrected stacking velocity exceeded the stacking velocity for horizontal reflectors by almost 40 percent at a dip of 60 degrees.

Levin's numerical results support analytically-based calculations of Byun (1984). Byun's insightful analysis addressed moveout of reflections from plane-dipping reflectors in transversely isotropic media under the assumption of small source-to-receiver 


\begin{tabular}{|c|c|c|c|c|c|c|}
\hline Medium & $\begin{array}{c}\sqrt{C / \rho} \\
\mathrm{m} / \mathrm{s}\end{array}$ & $\begin{array}{c}\sqrt{A / \rho} \\
\mathrm{m} / \mathrm{s}\end{array}$ & $\begin{array}{c}\sqrt{F / \rho} \\
\mathrm{m} / \mathrm{s}\end{array}$ & $\begin{array}{c}\sqrt{L / \rho} \mathrm{m} / \mathrm{s} \\
V_{A} / V_{C}\end{array}$ & $V_{\text {stack }}(0) / V_{C}$ \\
\hline Berea sandstone & 4206 & 4210 & 1961 & 2664 & 1.001 & 1.02 \\
Shale-limestone & 3306 & 3721 & 2076 & 1819 & 1.126 & 1.00 \\
Cotton Valley shale & 4721 & 5320 & 3095 & 2890 & 1.127 & 1.19 \\
Pierre shale & 2202 & 2235 & 1803 & 969 & 1.015 & 1.06 \\
\hline
\end{tabular}

Table 1. Velocity-type quantities and velocity ratios related to the four elastic moduli- $A, C, L$, and $F$, of White (1983) - that are pertinent to p-waves in transversely isotropic media. The four media listed are the same as those studied by Levin (1990). Here, $\rho$ is bulk density, $V_{A}=\sqrt{A / \rho}$ is $\mathrm{p}$-wave velocity in the horizontal direction, $V_{C}=\sqrt{C / \rho}$ is that in the vertical direction, $V_{L}=\sqrt{L / \rho}$ is s-wave velocity in the vertical direction, anf $V_{F}=\sqrt{F / \rho}$ is a velocity-like quantity that has no ready intepretation.

offset. While Byun's development allowed for multi-layered media, with homogeneous layers bounded by plane interfaces of arbitrary dip, his numerical examples pertained to just the case of a single homogeneous layer above a dipping reflector-the same model used by Levin.

While one may debate the nature of anisotropy present in the Earth's subsurface, without question the Earth is not homogeneous. In this study, I mimic Levin's 1990 studies of $V_{\text {stack }}(\phi)$ in media with vertical axis of symmetry, extending his approach to treat media that are vertically inhomogeneous as well as transversely isotropic. To limit the endless possibilities for ways in which an anisotropic medium can be vertically inhomogeneous, I follow Červený (1989) in considering only media for which the ratios among the various elastic moduli are independent of position. Cervený calls such media factorized anisotropic inhomogeneous (FAI). Yet another restriction placed in the media considered here is that velocity varies only linearly with depth.

Despite having lifted the restriction that media be homogeneous, the limitations on the media that will be studied here remain considerable. Justifications for these restrictions are varied. Primarily, as Shearer and Chapman (1988) show, the restriction to FAI media with linear velocity variation with depth affords simplicity and efficiency in the required traveltime computations. Beyond that, given the meager information available about elastic moduli for the subsurface, consideration of only FAI media with vertical variation seems a reasonable way to restrict the endless possibilities for inhomogeneous behavior. The choice of vertical axis of symmetry is based on Levin's result that anisotropy exerts little influence on stacking velocity when the symmetry axis is perpendicular to the reflector, but considerable influence when the 
symmetry axis is vertical. Vertical axis of symmetry seems to be an end-case of likely geologic importance. In contrast, the restriction to media with constant velocity gradient has less geologic justification. Constant-gradient media, at least, can model sizeable velocity variation with depth.

As in Levin's studies, iteration is required to do the ray tracing between specified source and receiver positions and to model Snell's law reflection in anisotropic media. One difference, however, is that whereas raypaths are straight for homogeneous media, they are curved here. Nevertheless, for the transversely isotropic FAI media considered here, the two-point ray tracing, based on a method of Shearer and Chapman, requires no costly numerical integration.

Our interest in stacking-velocity behavior stems from implications for quality of CMP-stacked data following either normal-moveout (NMO) or dip-moveout (DMO) correction. Routinely, DMO is based on the assumption that the subsurface medium is homogeneous, and, increasingly, DMO is based on velocity $v$ increasing with depth $z$-so-called $v(z)$ DMO. In neither case, however, is anisotropy taken into account. The primary purpose of this study is to assess, at least for the models considered here, implications of ignoring anisotropy in DMO when the subsurface is transversely isotropic.

\section{SHEARER AND CHAPMAN RESULT}

Consider just two-dimensional (2-D) propagation along the reflection raypath connecting specified source and receiver locations on a horizontal surface. As illustrated in Figure 1, the planar reflector is at the base of a single layer in which velocity increases linearly with depth (as suggested by the shading in the figure). In progressing from (1) homogeneous, isotropic models, to (2) homogeneous, transversely isotropic ones, and then to (3) our FAI models, ray tracing and thus traveltime computation becomes progressively more difficult. Traveltimes of reflections from a dipping interface in homogeneous, isotropic media are obtained simply from the law of cosines. Levin (1971) showed that the resulting moveout within CMP gathers is exactly hyperbolic, with stacking velocity given by

$$
V_{\text {stack }}(\phi)=V_{\text {stack }}(0) / \cos \phi \text {. }
$$

As Levin (1990) shows, for anisotropic (but still homogeneous) media, an iterative search is required to find the raypath from a source at the surface to the reflector and back to a receiver at the surfact. First, a straight ray is sent in a trial direction from the source to the reflector. At the reflector, Snell's law is used to give the direction of the reflected ray. For anisotropic media, however, Snell's law does not directly relate the angles of incidence and reflection associated with the raypaths (i.e., the paths along which energy travels). Instead, it relates the angles of incidence and reflection of plane waves - so-called phase angles. In general, the direction along which energy travels differs from the travel direction for corresponding plane waves, but the two 

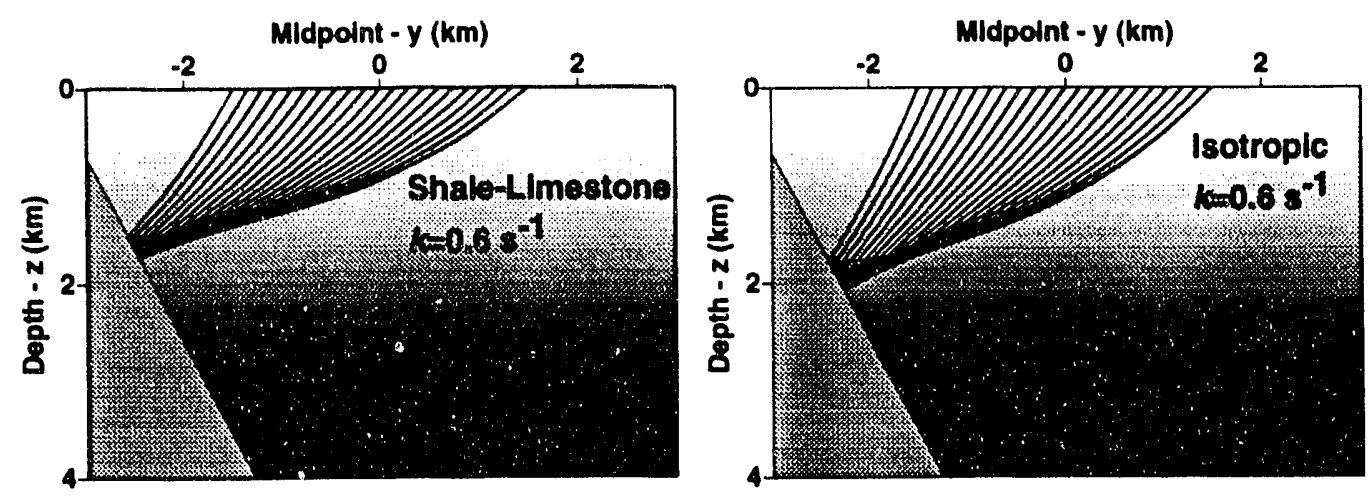

FIG. 1. Raypaths for reflections, in a CMP gather, from a dipping inic:sace beneath a medium with linear $v(z)$. The velocity function in the isotropic medium is identical to the vertical velocity function in the transversely isotropic shale-limestone medium listed in Table 1. Raypaths are circular for the isotropic medium, but not so for the shale-limestone. Note the differences in locations of reflection points.

directions are related to one another (Levin, 1990) and (Byun, 1984). These two authors describe the procedure for calculating the direction of the ray reflected from an interface:

- given the incident ray direction relative to the direction of the symmetry axis, compute the related incident plane-wave direction;

- given that plane-wave direction and the crientation of the reflector, use Snell's law to compute the reflected plane-wave direction; and

- from that computed plane-wave direction, compute the related direction of the reflected ray.

Levin points out that for anisotropic media, Snell's law gives a transcendental equation which is solved iteratively. The transcendental character comes from the fact that Snell's law involves both the direction and the velocity of the reflected plane wave, but that velocity is not known at the outset because it itself is a function of the as-yet-to-be-determined direction of the reflected wave.

For homogeneous media, once the direction has been determined for the reflected ray, the location where the ray intersects the horizontal surface is just the point of intersection of two straight lines. Recalling that this raypath initially left the source point in some trial direction, it is unlikely that the reflected ray that was just computed will intersect the surface at the desired receiver location. Following conventional two-point ray tracing, different take-off directions are then tried until the correct intersection point is found. The secant-search procedure (Press et al., 
1986, p. 248) generally converges within two to four iterations. Since the medium is homogeneous, traveltime along each straight leg of the raypati is just the quotient of the distance along the leg and the ray velocity in the direction of the ray.

When the medium is inhomogeneous as well as anisotropic, the procedure is much the same except that raypaths are now curved, so the ray tracing requires more computation than does just the intersection of two straight lines. For generally inhomogeneous media, this ray tracing requires numerical integration. Therefore, the integration step size decreases and, thus, the cost of the ray tracing increases with any increase in desired accuracy. I avert the bulk of the costly ray tracing with the assumption that velocity varies linearly in space.

For an isotropic medium with linear velocity variation, raypaths are just circles (Slotnick, 1959). Take-off ray directions, raypaths, and traveltimes are related analytically, so two-point ray tracing involves little more effort than that for straight raypaths. While raypaths and ray tracing are not so simple for anisotropic media, Shearer and Chapman (1988) have shown in an elegant proof that raypaths in FAI media with linear velocity variation can be simply described and, hence, efficiently computed. For our model of transverse isotropy with both symmetry axis and velocity gradient in the vertical direction, their result is as follows: raypaths in such media are merely scaled, 90-degree rotated versions of phase slowness (i.e., reciprocal of phase velocity) curves. Clearly, this property holds for isotropic media, for which both phase slowness curves and raypaths are circles.

For homogeneous, transversely isotropic media, phase velocity as a function of angle $\theta$ from the axis of symmetry is given by (White, 1983)

$$
\begin{aligned}
2 \rho v^{2}(\theta)= & (A+L) \sin ^{2} \theta+(C+L) \cos ^{2} \theta \\
& \pm\left\{\left[(A-L) \sin ^{2} \theta+(C-L) \cos ^{2} \theta\right]^{2}\right. \\
& \left.+4(L+F)^{2} \sin ^{2} \theta \cos ^{2} \theta\right\}^{\frac{1}{2}},
\end{aligned}
$$

where $A, C, F$, and $L$ are the four pertinent elastic moduli governing p- and sv-wave behavior. In equation (3), the plus sign in front of the radical pertains to p-waves, and the minus sign to sv-waves. We shall be interested in only p-waves here. Dividing both sides of equation $(3)$ by $2 \rho v^{2}(\theta)$ yields the slowness curve governing $2-\mathrm{D}$ wave propagation,

$$
\begin{aligned}
1= & 0.5\left\{(a+l) p_{1}^{2}+(c+l) p_{3}^{2}\right. \\
& +\left\{\left[(a-l) j_{1}^{2}+(c-l) p_{3}^{2}\right]^{2}\right. \\
& \left.\left.+4(l+f)^{2} p_{1}^{2} p_{3}^{2}\right\}^{\frac{1}{2}}\right\},
\end{aligned}
$$

where $a \equiv A / \rho$, etc., are the density-normalized elastic moduli, and

$$
p_{1}=\frac{\sin \theta}{v(\theta)}
$$




$$
p_{3}=\frac{\cos \theta}{v(\theta)}
$$

are the components of the phase slowness vector in the $x_{1}$ (horizontal) and $x_{3}$ (vertical) directions, respectively. $p_{1}$ is commonly called the ray parameter.

Now, consider an FAI medium in which all velocity quantities (e.g., $V_{C}=\sqrt{c}$ ) are linear functions of depth $z$. That is,

$$
\begin{aligned}
& V_{A}(z)=V_{A}(0)(1+g z) \\
& V_{C}(z)=V_{C}(0)(1+g z) \\
& V_{F}(z)=V_{F}(0)(1+g z) \\
& V_{L}(z)=V_{L}(0)(1+g z)
\end{aligned}
$$

and, in general,

$$
v(z, \theta)=v_{0}(\theta)(1+g z)
$$

where $V_{C}(0)$ is the vertical velocity at the surface, etc.; $g$ is the gradient factor, which is common to all velocity quantities in an FAI medium; $v(z, \theta)$ denotes the phase velocity in the $\theta$ direction at depth $z$; and $v_{0}(\theta)$ denotes that at the surface.

Following Shearer and Chapman (1988), let $x_{30} \equiv 1 / g$, and define a new, shifted vertical coordinate

$$
x_{3}=z+x_{30}
$$

Then, equation (7) becomes

$$
v\left(x_{3}, \theta\right)=v_{0}(\theta) g x_{3},
$$

and so on for the other velocity quantities.

Shearer and Chapman's remarkable result is that, for an FAI medium in which velocity varies as lescribed here, a scaling and 90-degree rotation of the slowness curve, equation (4), gives the following equation for a raypath characterized by the ray parameter $p_{1}$ in $x_{1}, x_{3}$ coordinates

$$
\begin{aligned}
\frac{2}{g^{2} p_{1}^{2}}= & \left(a^{0}+l^{0}\right) x_{3}^{2}+\left(c^{0}+l^{0}\right) x_{1}^{2} \\
& +\left\{\left[\left(a^{0}-l^{0}\right) x_{3}^{2}+\left(c^{0}-l^{0}\right) x_{1}^{2}\right]^{2}\right. \\
& \left.+4\left(l^{0}+f^{0}\right)^{2} x_{3}^{2} x_{1}^{2}\right\}^{\frac{1}{2}}
\end{aligned}
$$

In equation (10), the quantities $a^{0}, c^{0}, f^{0}$, and $l^{0}$ are the density-normalized, elastic moduli at the surface $z=0$.

Given a ray parameter $p_{1}$, to trace a ray trajectory I compute $x_{1}$ at different depths $z$ (or, equivalently, different vertical coordinates $x_{3}$ ). Squaring equation (10) gives a quadratic equation for $x_{1}^{2}$

$$
\alpha x_{1}^{4}+\beta\left(p_{1}, x_{3}^{2}\right) x_{1}^{2}+\gamma\left(p_{1}, x_{3}^{2}\right)=0,
$$


where the coefficients $\alpha, \beta$, and $\gamma$ all depend on the elastic moduli at the surface, as well as on $p_{1}$ and $x_{3}$. Figure 2 depicts a raypath in the $x_{1}, x_{3}$ coordinates. One of the four roots $x_{1}$ for a given $x_{3}$ is pointed out in the figure, and another is just at the arrow along the raypath to the right of the $x_{1}$ axis. The other two roots give positions along a raypath for sv-waves.

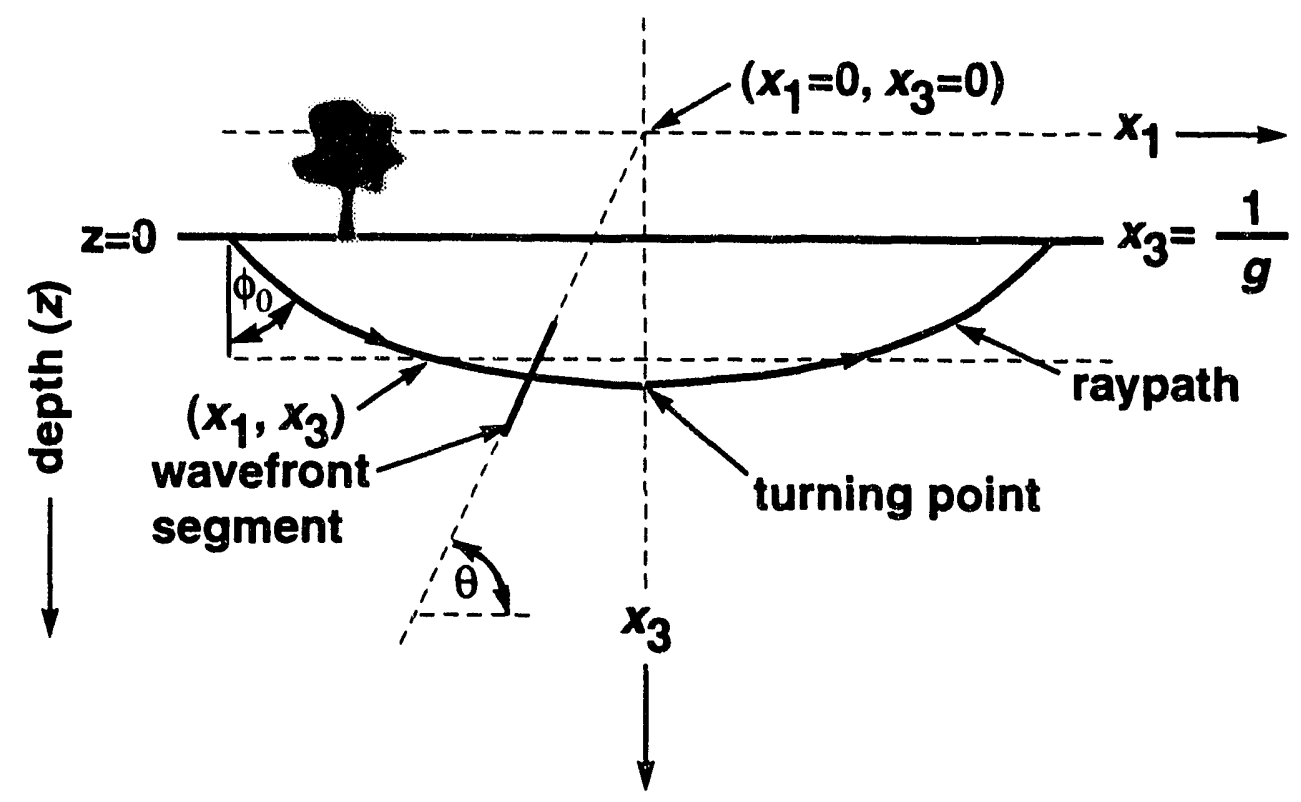

FIG. 2. Raypath in a linear $v(z)$, transversely isotropic FAI half-space, shown in $\left(x_{1}, x_{3}\right)$ coordinates. The medium's surface, $z=0$, is equivalent to $x_{3}=1 / g=V_{0} / k$. Also, $x_{1}=0$ at the turning point, i.e., $x_{1}=0$ is a line of symmetry for the raypath.

For an isotropic medium with the same linear $v(z)$ behavior, the moduli satisfy $a=c=f+2 l$, and equation (11) reduces to the familiar equation of a circle (Slotnick, 1986)

$$
x_{1}^{2}+x_{3}^{2}=\left(\frac{1}{g \sin \theta_{0}}\right)^{2}=\left(\frac{1}{g V_{0} p_{1}}\right)^{2},
$$

where $V_{0}$ is now independent of $\theta$.

From equation (10), full description of the transversely isotropic FAI medium requires just $V_{C}(z)$ and the three ratios $a / c, f / c$, and $l / c$. For the studies below, I write the linear $V_{C}(z)$ behavior slightly differently than in equation (6), as follows:

$$
V_{C}(z)=V_{0}+k z
$$

where, clearly $k=g V_{0}$, and, for shorthand, $V_{0}$ is the vertical velocity at the surface $z=0$.

\section{TRAVELTIME COMPUTATION}

While it is of interest to see the curved raypaths that result from the ray tracing (e.g., those in Figure 1), our true interest is in computation of traveltimes between 
specified points on the raypaths. Here, I follow Cervený (1989), who gives a comprehensive discussion of general FAI media along with a particularly lucid summary of the Shearer and Chapman result. Cervený shows that the vertical component of slowness $p_{3}$ behaves as follows

$$
p_{3}(u)=p_{3}\left(u_{0}\right)-(g)\left(u-u_{0}\right)
$$

where $u$ is a variable along the ray, related to traveltime, and $u_{0}$ is the value of that variable at the starting point of the raypath. He also shows that the traveltime along the raypath requires the numerical integration

$$
\tau_{3}(u)=\tau_{3}\left(u_{0}\right)+\int_{u_{0}}^{u} g x_{3}(u) d u .
$$

The 90-degree rotation of the slowness curve to obtain the raypath was based on the observation that in the $x_{1}, x_{3}$ coordinate system, the components of slowness along a raypath must satisfy

$$
p_{1} x_{1}+p_{3} x_{3}=0
$$

That is, the position vector $\mathbf{x}$ and the slowness vector $\mathbf{p}$ are orthogonal everywhere along a raypath within such a medium. This is obviously true for the circular raypaths in isotropic media, but not so readily apparent for anisotropic media. Recall that, in anisotropic media, the ray and phase directions differ, so that raypaths are not generally normal to wavefronts (see Figure 2). Phase directions and wavefronts, however, are normal to one another.

Eliminating $p_{3}$ from equations (14) and (16) and setting the arbitrary value $u_{0}$ to zero gives

$$
x_{1}=\left(\frac{x_{3}}{p_{1}}\right)\left(\frac{u}{g}-p_{30}\right) .
$$

Substituting this expression for $x_{1}$ in equation (11) gives another quadratic equation, this one giving $x_{3}^{2}(u)$, which provides the integrand for equation (15).

Thus, although numerical integration is required to compute traveltime, that integration is straightfr rward. I use the efficient scheme of Press et al. (1986, p. 110) for integration by the extended trapezoidal rule.

\section{TWO-POINT RAY TRACING FOR REFLECTION RAYPATHS}

In ray-trace modeling, we generally wish to compute the path and traveltime between specified source and receiver positions in some $x, z$ coordinate system. Therefore, to use the method described above, it is necessary to relate the $x_{1}, x_{3}$ and $x, z$ coordinates to one another. Equation (8) gives the simple translation required for the vertical coordinates. We can express the required iranslation for the lateral coordinate transformation as $x_{1}=x-x_{c}$, where $x_{c}$ is the coordinate corresponding to the origin in $x_{1}$. To get at $x_{c}$, consider a ray from some point $x_{s}, z_{s}$ to its intersection with 
depth $z_{r}$. The raypath is characterized by a fixed ray parameter $p_{1}$, and that fixed value also fixes the location of $x_{c}$. With $p_{1}$ given, I solve equation (11) twice, once with depth $x_{3}=x_{3 s}$ and again with $x_{3}=x_{3 r}$. The $x$ coordinate $x_{r}$ of the raypath at depth $z_{r}$ is then given by

$$
x_{s}+\left(x_{1 r}-x_{1 s}\right)=x_{s}+\left(x_{r}-x_{c}\right)-\left(x_{s}-x_{c}\right)=x_{r} .
$$

We can now address two-point ray tracing along a reflection path, such as in Figure 1, between a specified source and receiver location. For all but the simplest models, in two-point ray tracing one does not know at the outset the value of the ray parameter that describes the path connecting the two points. Therefore, starting with an educated guess for the value of the ray parameter, some systematic search procedure is required to arrive at the correct value. Such is also the case here.

In principle, we would first solve the appropriate equations that give the intersection of a downward ray from the source (specified by a trial ray parameter, or equivalently, trial starting direction) with the dipping reflector. For the linear $v(z)$, transversely isotropic FA.I medium considered here, computation of that intersection point would require determination of the correct root of a quartic equation for $x_{3}$. Rather than working with quartic equations, I follow the two-step search procedure illustrated in Figure 3.

Instead of solving directly for the location of a trial reflection point $R_{t}$ along the dipping reflector (Figure 3a), I first specify a trial depth $z_{t}$ for the reflection-point position and a trial ray parameter. The point $x_{t}$ at which the trial raypath arrives at depth $z_{t}$ is the solution of quadratic equation (11). A secant search then finds the ray parameter that yields $x_{t}$, the lateral coordinate of $R_{t}$.

At the trial reflection point (Figure 3b), Snell's law gives the starting direction for the upward portion of the raypath. For anisotropic media, Snell's law involves a relationship between the phase angles and phase velocities, which themselves are functions of the phase angles. The incident phase angle at the interface is readily obtained from equation (16) (see also, Figure 2); that is,

$$
\frac{p_{1}}{p_{3}}=-\frac{x_{3}}{x_{1}}=\tan \theta
$$

where, again, $\theta$ is phase angle with respect to vertical. Given this angle and the reflector dip $\phi, 1$ follow the iterative solution of the transcendental Snell's law equation described by Levin (1990) to find the reflection ray angle. Once that angle is determined, equation (19) then yields the ray parameter for the upward path.

Again, I solve quadratic equation (11), this time to get the location $e$ of the intersection of the upward path with the surface $z=0$. Since, in general, this point will miss the desired receiver location, I do a second stage of secant search by varying the trial depth $z_{t}$ of the reflection point (Figure 3c), and thus continue to vary $z_{t}$ and $p_{1}$ until $e \approx g$, the location of the receiver. 

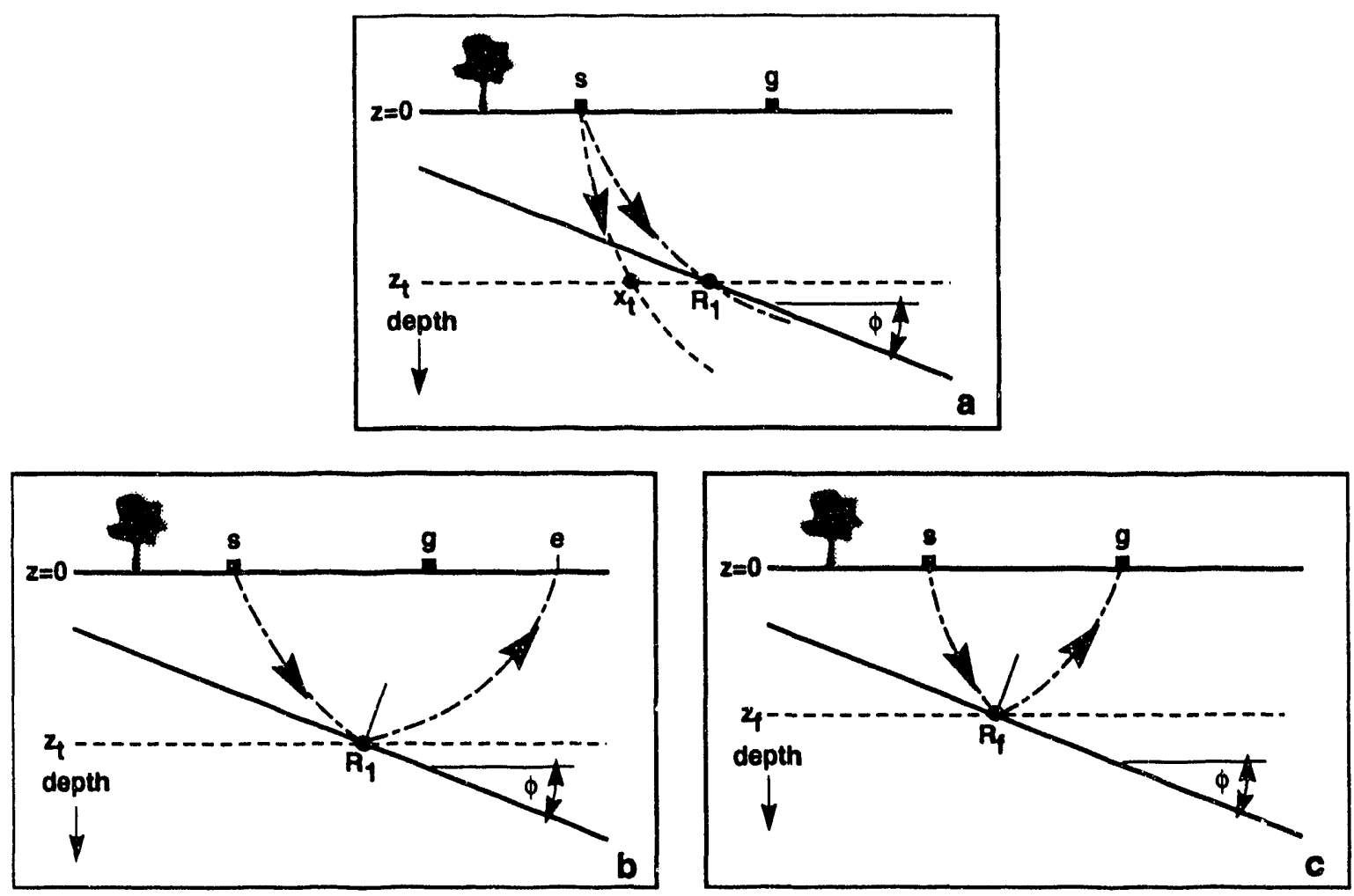

FIG. 3. Steps in the two-point ray tracing to obtain the raypath from a source to a dipping reflector and back up to a receiver. (a) Solve a quadratic equation for the lateral coordinate $x_{t}$ at which the downward ray with trial ray parameter $p_{t}$ intersects trial depth $z_{t}$. Then do a secant search to find the ray parameter for the raypath to the trial reflection point $R_{t}$ at depth $z_{t}$ along the reflector. (b) Apply Snell's law (for transverse isotropy) to find the ray parameter for the reflected portion of the raypath. This path intersects the surface at $e$, which differs from the desired receiver location $e$. (c) Do another secant search to find the depth of the reflection point and the ray parameter for the downward portion of the reflection raypath to the receiver.

\section{MOVEOUT BEHAVIOR}

In his studies with the four homogeneous media listed in Table 1, Levin (1990) found that for spreadlength $X_{\max }$ comparable to the distance from the CMP to the dipping interface, moveout was approximately hyperbolic for even the most anisotropic of the four media - the shale-limestone. While we expect the hyperbolic approximation to hold for small angles even when the medium is both anisotropic and inhomogeneous, it is of interest to know if that approximation can also hold for routinely used spreadlengths (i.e., spreadlength comparable to distance from CMP to reflection point).

Figure 4 is a plot of $T^{2}$ versus $X^{2}$, where $T(X)$ is reflection time at offset $X$, for the shale-limestone, with different choices of velocity gradient $k$ (see equation [13]). For all cases, the root-mean-square (rms) vertical velocity down to $z=3000 \mathrm{~m}$ is 
$3306 \mathrm{~m} / \mathrm{s}$, the value for $V_{C}$ used by Levin, and the spreadlength equals $3000 \mathrm{~m}$, the distance from the CMP to the reflector. While nowhere in the Earth's subsurface is velocity variation simply linear, the displayed range of $k$ values modeled spans the spectrum from homogeneous media to media that are highly variable with depth. $k=1.2 \mathrm{~s}^{-1}$ is quite large, and $k=0.6 \mathrm{~s}^{-1}$ is a fairly representative characterization of the subsurface.

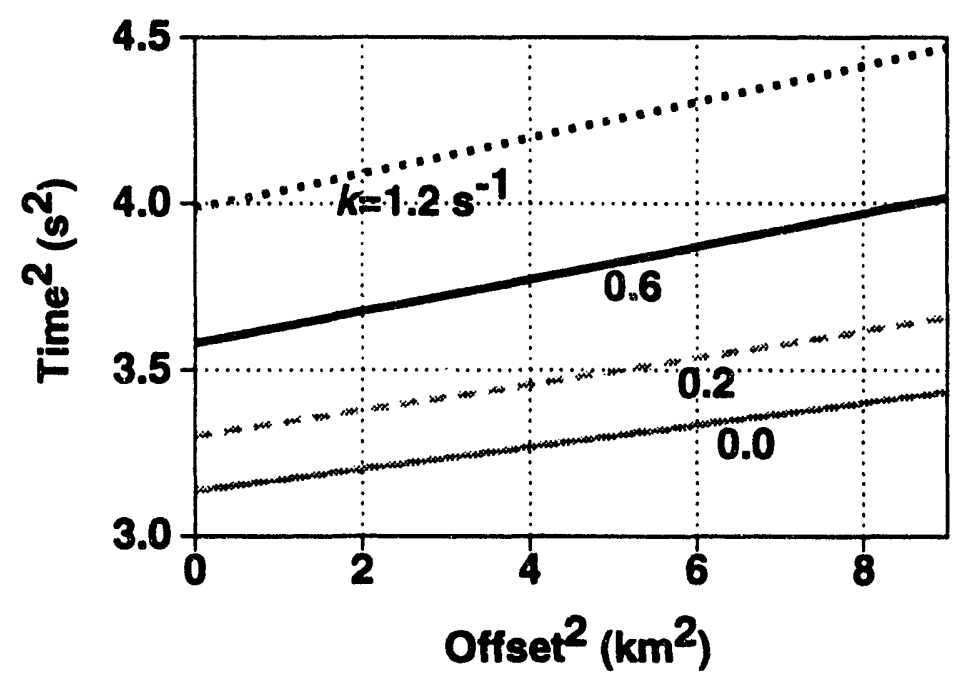

FIG. 4. $T^{2}-X^{2}$ curves for reflections from a dipping reflector (dip $=40$ degrees) beneath various media with the transversely isotropic properties of the shale-limestone. The four media, all of which have the same rms velocity between the si-face and depth $=3000 \mathrm{~m}$, differ only in the velocity gradient $k$.

All curves in Figure 4 are approximately linear, indicating that the hyperbolic assumption continues to be valid for our linear $v(z)$, transversely isotropic models. We shall consider this assumption more closely below.

The initial purpose of this study was to extend Levin's numerical studies of stacking velocity to media that are inhomogeneous, as well as transversely isotropic. Figure 5 is an independent reproduction of the dip-corrected stacking velocities in Figure 3 of Levin (1990). Stacking velocity was computed from a least-squares linear fit to the $T^{2}-X^{2}$ moveout data over a spreadlength of $3000 \mathrm{~m}$; then the velocities were "dip-corrected" by multiplication with $\cos \phi$ in accordance with equation (2). For a homogeneous, isotropic medium, this dip correction should bring stacking velocity for all dips to the value for zero dip. This is just what DMO would accomplish when applied to data from such a model. Byun (1984) did this same correction, calling the corrected stacking velocity diffractor velocity.

From the variation of the dip-corrected curves with dip in Figure 5, we would infer that the shale-limestone is the "most anisotropic" of the four media tested, 


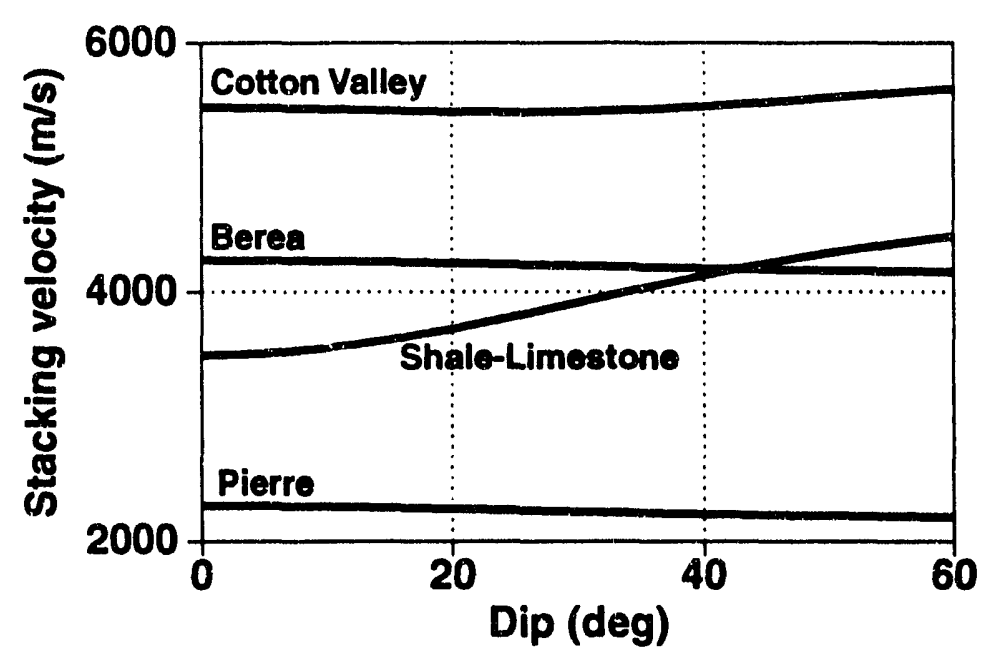

FIG. 5. Cosine-of-dip corrected stacking velocity as a function of reflector dip for the four homogeneous, transversely isotropic media in Table 1. These results duplicate those of Levin (1990). The distance from the CMP to the reflector is $3000 \mathrm{~m}$ in all cases.

with the Cotton Valley shale a distant second, followed by the Pierre shale and Berea sandstone. Also, as observed by Levin, the variations exhibit no simple or predictable behavior with dip. The difference in behavior of the curves for the shale-limestone and the Cotton Valley shale is particularly noteworthy since the ratios of horizontal to vertical velocity of these two media are almost identical (see Table 1). As mentioned above, this failure of the ratio $V_{A} / V_{C}$ as a predictor of much anisotropic behavior was explained by Thomsen (1986), who showed, for homogeneous media, that the ratio of normal-moveout velocity to vertical velocity had no relationship whatsoever to $V_{A} / V_{C}$. The values computed for $V_{\text {stack }}(0) / V_{C}$ tabulated in Table 1 quantitatively match Thomsen's predictions. Moreover, for all values of $k \neq 0$ studied here, the values in column 7 of that table are unchanged when $V_{C}$ is replaced by the vertical rms velocity to the reflector.

Now, consider inhomogeneous media of the type treated in this paper. Figure 6 shows dip-corrected stacking velocity as a function of reflector dip for several variants of the same four media treated by Levin. By a variant of a given medium (e.g., shalelimestone), I mean that the ratios among all the elastic moduli at a given depth are the same as those obtained from the moduli for the (shale-limestone, in this example) medium listed in Table 1, independent of the gradient $k$. Superimposed in Figure 6 are curves for media with velocity gradients $k=0$ (Levin's homogeneous media), and $k=0.2,0.6$, and $1.2 \mathrm{~s}^{-1}$. The results show a remarkable insensitivity of dip-corrected stacking velocity to the degree of inhomogeneity. The only noticeable tendency is 
for an increase in velocity gradient to reduce the dip-dependence of the dip-corrected velocities for the shale-limestone. Thus, the inhomogeneity has a benign influence on the curves, mitigating somewhat the stacking-velocity variations introduced by the anisotropy. This general tendency of $v(z)$ to reduce the influence of anisotropy was observed in a concurrent study of migration-velocity error as a function of reflector dip (Larner and Cohen, 1992).

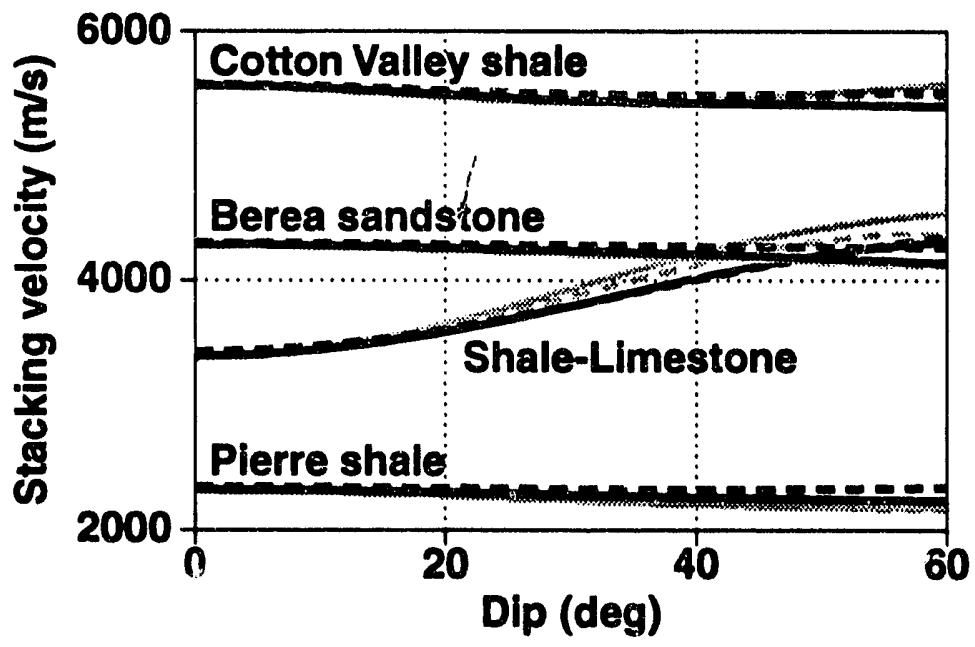

FIG. 6. Dip-corrected stacking velocity as a function of reflector dip for inhomogeneous variants of the same four transversely isotropic media shown in Figure 5. The velocity gradients for the solid gray, dashed gray, solid black, and dashed black curves are, respectively, $k=0.0,0.2,0.6$, and $1.2 \mathrm{~s}^{-1}$.

Two points of clarification about the curves in Figure 6 are appropriate. First, the vertical velocity at the surface differs for the media with different gradients in such a way that the rms velocity down to the reflector depth is the same for all variants and is equal to the value of $V_{C}$ listed in Table 1 . Any other choice would have resulted in a large separation of the curves for the different gradients in Figure 6. The similarities among the curves for the variants of any one of the four media shown in that figure thus supports a working hypothesis that has been of much value in analysis and processing of isotropic data: the reflection-time behavior for $v(z)$ media can be largely predicted through modeling with a homogeneous model having velocities comparable to the rms velocities in the inhomogeneous medium. Examples of this are the so-called straight-ray assumption and the adequacy of applying Kirchhoff migration with use of rms velocities to image data where reflectors are not too steep. Thus, we can use simple homogeneous models, such as those in Levin's papers, for study of some anisotropic phenomena in models where the true medium velocity actually varies with depth. 
The second point relates to the nature of the dip correction. For homogeneous media. the dip correction to apply to stacking velocity is just the cosine of the reflector dip. For $v(z)$ media, however, the dip correction is more complicated and differs substantially from $\cos \phi$, while still ignoring anisotropy. The $v(z)$ dip correction is obtained from an analytic expression for the small-offset velocity (or NMO velocity) $V_{n m o}$ associated with reflections from a dipping interface beneath a linear $v(z)$ medium.

Shah (1973) and Hubral and Krey (1980) have shown the direct relationship that in general exists between NMO velocity and wavefront curvature near the location where the normal-incident ray intersects the surface. For small offset, NMO velocity is given approximately by

$$
V_{n m o}^{2}=\frac{1}{T_{0}} \frac{d X}{d p}
$$

where $T_{0}$ is the normal-incidence reflection time, $X$ is source-to-receiver offset, and $p$ is the ray parameter, which I previously called $p_{1}$. For $v(z)$ media, $X(p)$ is given by

$$
X(p)=\int_{0}^{z_{r}} \frac{p v(\sigma)}{\sqrt{1-p^{2} v^{2}(\sigma)}} d \sigma
$$

where $z_{r}$ is the depth at the normal-incidence reflection point. Differentiating gives

$$
\frac{d X}{d p}=\int_{0}^{z_{r}} \frac{v(\sigma)}{\left[1-p^{2} v^{2}(\sigma)\right]^{3 / 2}} d \sigma
$$

Also, $T_{0}$ satisfies

$$
T_{0}(p)=\int_{0}^{z_{r}} \frac{d \sigma}{v(\sigma) \sqrt{1-p^{2} v^{2}(\sigma)}} .
$$

Evaluation of these integrals for $v(z)=v_{0}+k z$ gives

$$
V_{n m o}^{2}(\phi)=\frac{v_{0}^{2}\left(\cos \theta_{0}-\cos \phi\right)}{\sin ^{2} \theta_{0} \cos \theta_{0} \cos \phi \log \left[\frac{v\left(z_{r}\right)}{v_{0}} \frac{1+\cos \theta_{0}}{1+\cos \phi}\right]}
$$

Here, I have used the fact that for the zero-offset path, $\theta(z)=\phi$ in an isotropic medium. For reflections from a horizontal reflector (i.e., the normal-incidence path is vertical), similar evaluation of the appropriate integrals gives

$$
V_{n m o}^{2}(0)=\frac{v^{2}\left(z_{r}\right)-v_{0}^{2}}{2 \log \left[\frac{v\left(z_{r}\right)}{v_{0}}\right]}
$$

The dip-correction factor $h(\phi)$ applied to the stacking velocities in Figure 6 and in subsequent figures is then

$$
h(\phi)=\frac{V_{n m o}(0)}{V_{n m o}(\phi)} .
$$


For a homogeneous medium, this factor reduces to $\cos \phi$.

\section{IMPLICATIONS FOR DIP-MOVEOUT}

The importance of applying the dip correction $h(\phi)$ and the large difference between it and $\cos \phi$ are suggested in Figure 7. Figure 7a shows three curves of stacking velocity versus dip for the shale-limestone with the "representative" velocity gradient, $k=0.6 \mathrm{~s}^{-1}$. The gray curve has had no correction for dip; it is directly the velocity that gives the best fit to moveout over a spreadlength. The black curve has the $h(\phi)$ correction, and the dotted curve has a correction that is close to, but not quite, $\cos \phi$. The correction used in the dotted curve (same as the black curve for shale-limestone in Figure 6 ) is $\cos \hat{\phi}$, where $\hat{\phi}$ is the apparent dip as perceived by a constant-velocity DMO process. It is given by

$$
\sin \hat{\phi}=p V_{\text {stack }}(0) \text {. }
$$

That is, the apparent dip is the value that one would compute under the simplistic assumption that the subsurface is homogeneous with velocity equal to the observed stacking velocity for horizontal reflectors. Constant-velocity DMO makes this same simplistic assumption.
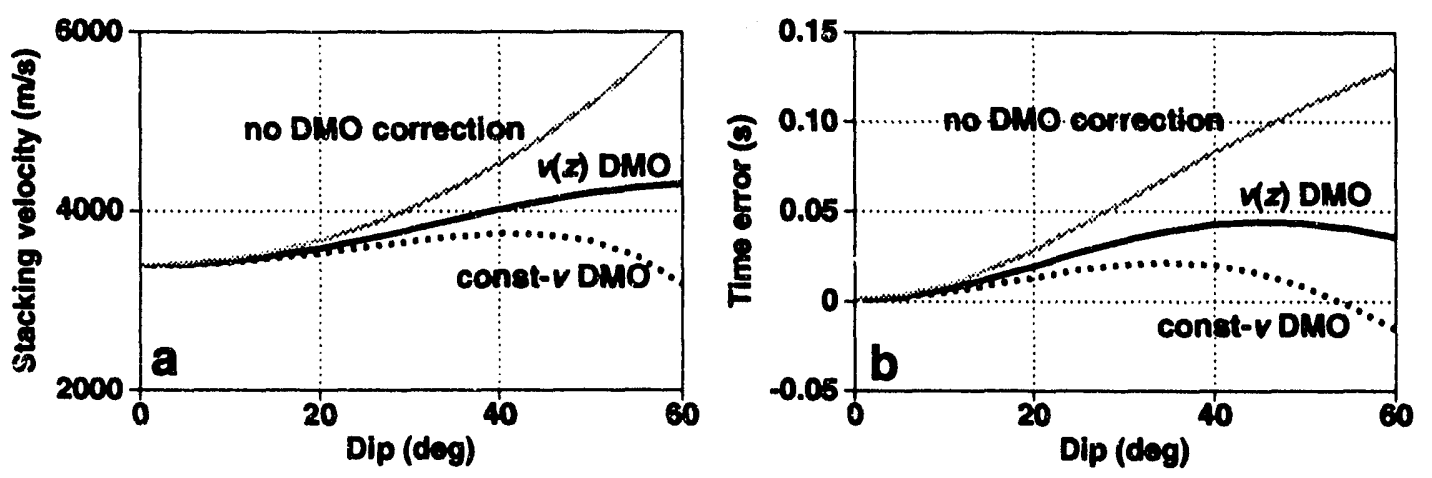

Fig. 7. (a) Stacking velocity as a function of reflector dip for the shale-limestone medium with velocity gradient $k=0.6 \mathrm{~s}^{-1}$-uncorrected for dip (gray), after DMO correction with a $v(z)$ DMO process (black), and after DMO correction with a constant-velocity DMO process (dotted). (b) Residual moveout $\Delta t$ at offset $X=3000 \mathrm{~m}$ as a function of reflector dip for the same shale-limestone data uncorrected (gray), DMO corrected with $v(z)$ DMO (black), and DMO corrected with constant-velocity DMO (dotted).

Clearly, from Figure 7a, either of the dip corrections brings the corrected stacking velocity closer to the desired zero-dip velocity than does no correction. It appears, in fact, that for the shale-limestone, the most anisotropic of the four media studied, the constant-velocity DMO actually would perform better than a $v(z)$ DMO (recall that both ignore anisotropy; as a result neither properly corrects the stacking velocities). 
Beyond the qualitative observations on relative behavior of the velocity curves, we should be concerned with just how important are these differences in stacking velocity. Errors in stacking velocity leave primary reflections misaligned across individual CMP gathers and hence cause those primaries-particularly the higher-frequency components - to be attenuated when the data are stacked. Therefore, ultimately it is this misalignment that should be of most concern.

Let us postulate that, as is routinely done in practice, data receive an NMO correction, with or without DMO, that is based on the observed stacking velocity for horizontal reflections. Then, since the moveout of reflections from dipping reflectors differs from this NMO, reflections will be misaligned prior to CMP stacking. With the stacking velocities measured in our study, we can quantify the resulting timing misalignment. Our measure of misalignment, which I shall call time error or residual moveout, is the difference in time that would arise on the far-offset trace. In these tests, the largest offset is $3000 \mathrm{~m}$, comparable to the distance to the reflector from the CMP.

Plotted in Figure $7 \mathrm{~b}$ are the time errors that arise when the data are simply NMO corrected (no dip correction) and when they are DMO corrected with either a constant-velocity DMO or a $v(z)$ DMO. Timing errors are reduced substantially when either of the (isotropic) DMO algorithms is used, but neither of the DMO approaches reduces the error as much as would be desired. Also, note that although the medium is inhomogeneous, the constant-velocity $D^{*} \cup$ correction yields the better result of the two. As we shall see, this is a fortuitous result peculiar to the shale-limestone medium.

The dependence of these moveout errors on velocity gradient for the shale-limestone is exhibited in Figure 8. The curves for $k=0.6 \mathrm{~s}^{-1}$ are identical to those in Figure $7 \mathrm{~b}$. In addition, Figure 8 shows curves for a larger gradient, $k=1.2 \mathrm{~s}^{-1}$, and for a homogeneous medium. For the homogeneous medium, $k=0$, constant-velocity DMO and $v(z)$ are identical; both involve just the $\cos \phi$ correction. Note that the larger the velocity gradient, the less serious is the residual moveout when the data are just NMO-corrccted. Also, while the errors that arise when constant-velocity DMO is applied vary considerably with velocity gradient, those that arise when $v(z)$ DMO correction is done are less sensitive to the gradient.

The curves in the bottom right portion of the figure pertain to an isotropic medium with velocity gradient of $k=0.6 \mathrm{~s}^{-1}$. Since ignoring isotropy in DMO is no issue for these data, the residual moveout goes to nearly zero when the correct $v(z)$ DMO is applied. If, instead, constant-velocity DMO is applied, the data become overcorrected for the errors that arise when NMO alone is performed. All of the linear $v(z)$ models studied exhibit this known tendency for the constant-velocity DMO to overcorrect data from isotropic models. Also, in all cases the error after over-correction is smaller than the error when no DMO is applied. In other words, for isotropic, linear $v(z)$ media, applying constant-velocity DMO always yields improvement over not doing DMO. 

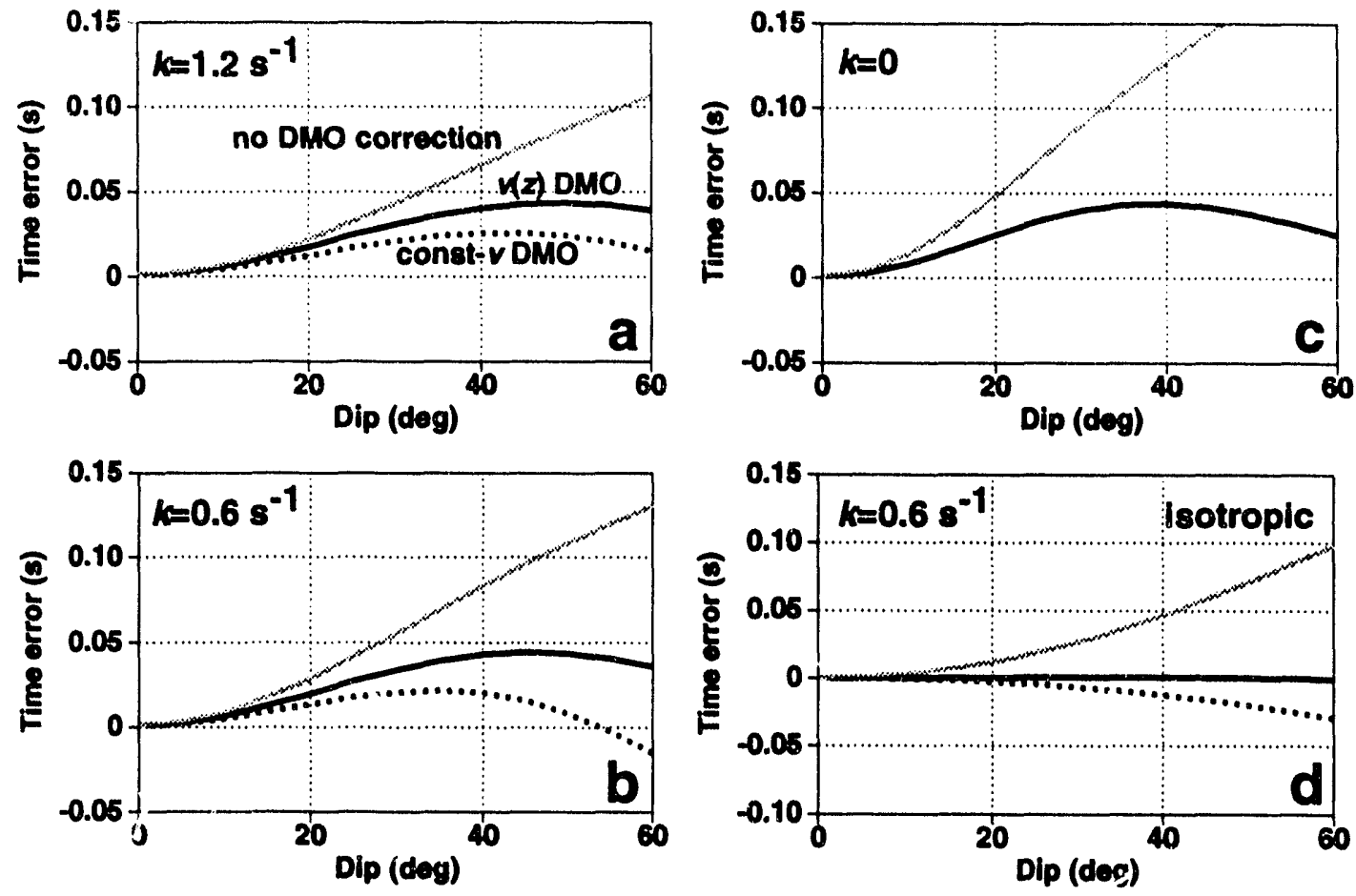

FIG. 8. Residual moveout $\Delta t$ at offset $X=3000 \mathrm{~m}$ as a function of reflector dip for variants of the shale-limestone-data uncorrected for dip (gray), DMO corrected with $v(z)$ DMO (black), and DMO corrected with constant-velocity DMO (dotted). In (a), (b), and (c), the medium is transversely isotropic with velocity gradient $k=1.2,0.6$, and $0.0 \mathrm{~s}^{-1}$, respectively. In (d) the gradient is again $k=0.6 \mathrm{~s}^{-1}$, but here the medium is isotropic. Note that data from the isotropic medium are well corrected with a $v(z)$ DMO correction. Also, in (c), since the medium is homogeneous, $v(z)$ DMO is identical to constant-velocity DMO.

Similar curves of residual moveout error for the four media-shale-limestone, Cotton Valley shale, Berea sandstone, and Pierre shale-are compared in Figure 9. Here, we see that, in terms of expected DMO performance, the shale-limestone on which we have focused most of our attention is the most anomalous, indeed the most anisotropic, of the four media. $v(z)$ DMO corrects the timing misalignment for the other three media almost as well as if they were isotropic.

Based on the residual moveout after $v(z)$ DMO in Figure 9, the anisotropy of the Pierre shale appears to be a distant second to that of the shale-limestone, with the Cotton Valley shale and Berea appearing to be only weakly anisotropic. This ranking of the relative "degree of anisotropy" among the four media differs somewhat from that estimated on the basis of the curves in Figures 5 and 6, where the Cotton Valley shale showed larger variation in stacking velocity with dip than did the Pierre shale. The variability of the stacking velocities, however, is misleading. Since the 
Cotton Valley shaie is a much higher-velocity medium than is the Pierre shale, a given moveout error would give rise to a larger variation in velocity for the Cotton Valley than for the Pierre. For this reason, the behavior of residual timing errors likely is the more appropriate measure of the relative importance of anisotropy in DMO correction.
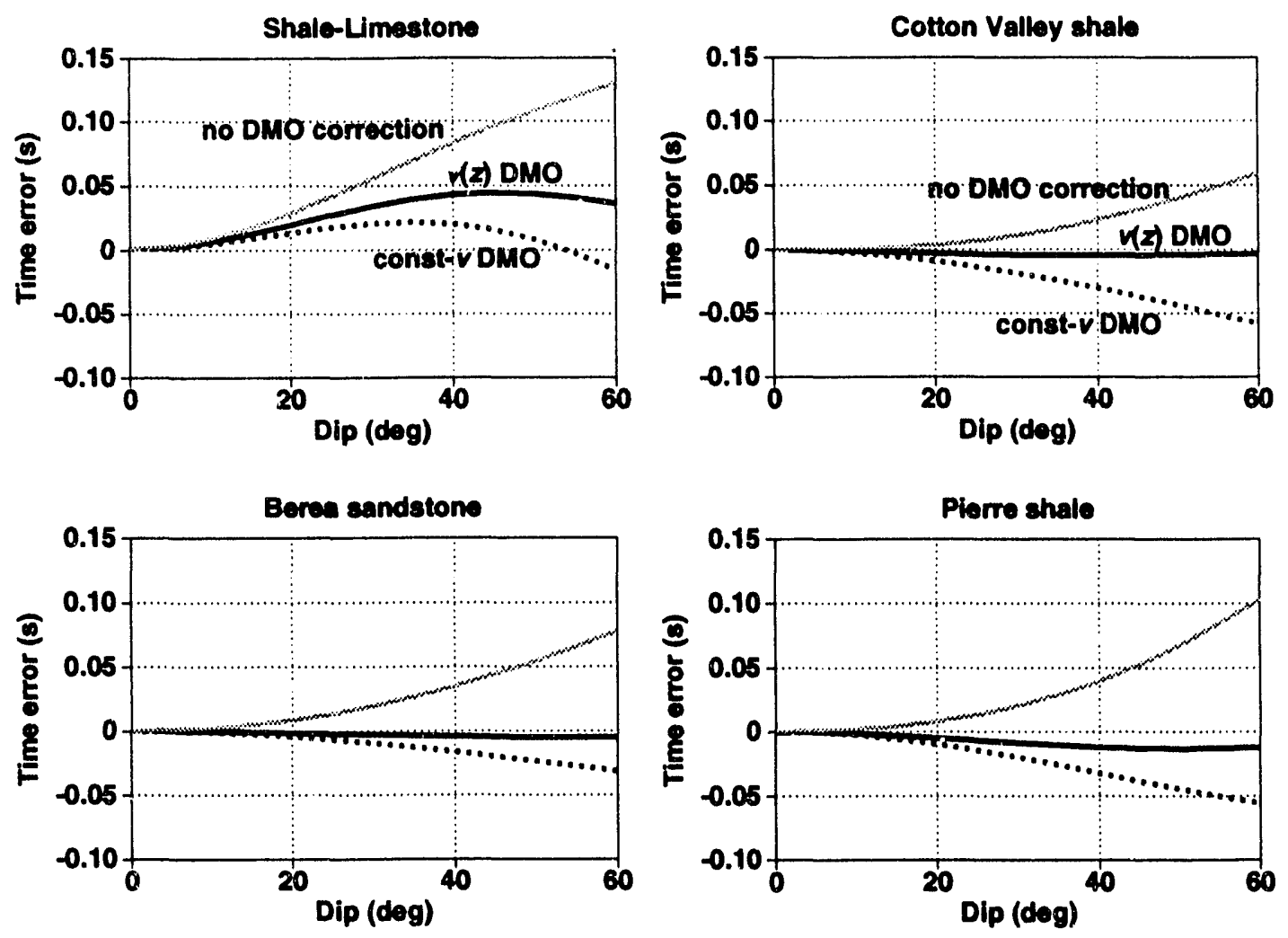

Fig. 9. Residual moveout $\Delta t$ at offset $X=3000 \mathrm{~m}$ as a function of reflector dip for four media-data uncorrected for dip (gray), DMO corrected with $v(z)$ DMO (black), and DMO corrected with constant-velocity DMO (dotted). The vertical velocity gradiant is $k=0.6 \mathrm{~s}^{-1}$ in all cases. Note that $v(z)$ DMO with anisotropy ignored is adequate not only for the weakly anisotropic Berea sandstone but also for the considerably anisotropic Cotton Valley shale.

\section{NORMAL-MOVEOUT VELOCITY VERSUS STACKING VELOCITY}

As seen in Figure 4, data from our transversely isotropic, FAI linear $v(z)$ models all have moveout that is well approximated by hyperbolas for spreadlengths typically used in practice. This suggests that an efficient alternative to hyperbolic fitting of traveltimes to obtain the stacking velocity $V_{\text {stack }}$ would be the evaluation of an analytic expression for the small-offset moveout velocity, which I earlier called $V_{n m o}$ 
(see equation [24]). The relative simplicity of the ray-tracing equations for linear $v(z)$, isotropic media allowed us to derive the analytic expression in that equation.

For our transversely isotropic FAI media, also with linear $v(z)$, it happens that we can also obtain an analytic expression for $V_{n m o}$ once we have done the ray tracing for the zero-offset raypath. Whether or not a medium is isotropic, $V_{n m o}$ can always be written as in equation (20). The ray tracing for the zero-offset raypath provides the measured normal-incidence traveltime $T_{0}$, so we require an expression for the derivative $d X / d p$. Equation (11) gives an expression involving $x_{1}$ as a function of $p$, which I called $p_{1}$ there. Differentiating that equation with respect to $p$ gives

$$
\frac{d x_{1}}{d p}=-\frac{x_{1}^{2} \frac{d \beta}{d p}+\frac{d \gamma}{d p}}{2 x_{1}\left(2 \alpha x_{1}^{2}+\beta\right)}
$$

All the quantities on the right-hand side in equation (28) are known from the ray tracing along the normal-incidence ray. The desired derivative $d X / d p$ is given by

$$
\frac{d X}{d p}=\frac{d x_{10}}{d p}-\frac{d x_{1 r}}{d p}
$$

where $x_{10}$ is the value of $x_{1}$ at the surface of the medium, and $x_{1 r}$ is the value of $x_{1}$ at the reflector. Of course, when sources and receivers change within a CMP gather, the reflection point at a dipping reflector moves as well. Holding the reflection point fixed in this computation is justified on the basis of Fermat's principle, which implies that the traveltime from source to reflector to receiver is stationary at the true reflection point.

Figure 10 compares $V_{\text {stack }}$, based on the hyperbolic fit over a spreadlength, with $V_{n m o}$, computed from equations (29), (28), and (20). The curves show stacking velocity, uncorrected for dip, for the four media with gradient $k=1.2 \mathrm{~s}^{-1}$. The match is excellent for the three media other than the shale-limestone. While the match is good for the shale-limestone, perceptible differences between the two types of velocity suggest that the hyperbolic assumption is violated to some extent for some dips. These differences are an indication that the $T^{2}-X^{2}$ curves are not quite as straight for the shale-limestone medium as are those for the other media. (The differences are smaller for smaller values of $k$-not shown here.)

\section{CONCLUSION}

The behavior of dip-corrected stacking velocity with reflector dip computed by Levin holds with remarkably little change when vertical inhomogeneity is incorporated into the model of transverse isotropy, as long as the dip-correction is appropriate to $v(z)$ media. Without the dip correction, the larger the velocity gradient the smaller is the residual moveout that exists in NMO-corrected data. For the media considered here, stacking-velocity dependence on reflector dip can be adequately investigated 


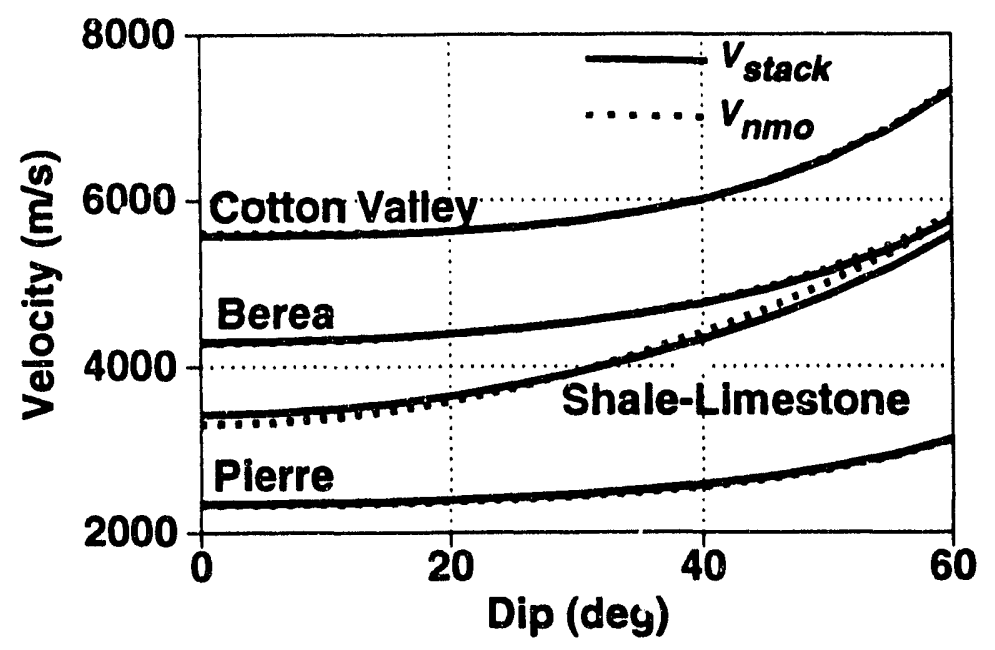

FIG. 10. Comparison of stacking velocity (solid) and normal-moveout velocity (dashed) - both uncorrected for dip--as a function of reflector dip, for the four media under study. Stacking velocity was computed by least-squares fitting of a straight line to $T^{2}-X^{2}$ over an offset distance of $X=3000 \mathrm{~m}$, while the normal-moveout velocity is based on small-offset wavefront curvature. The largest departures are for the shale-limestone, indicating that the hyperbolic approximation for moveout for that medium is not valid for such large offset $X$. Here, $k=1.2 \mathrm{~s}^{-1}$.

with homogeneous models having velocities that equal the counterpart rms velocities of the inhomogeneous media.

Only one of the four media studied - the shale-limestone- exhibits dramatic influence of anisotropy on stacking-velocity behavior. This, despite the fact that, in other respects, two of the other media-Cotton Valley shale and Pierre shale-are considered to be equally or more anisotropic than the shale-limestone. For example, the ratio of horizontal to vertical velocity in the Cotton Valley shale is almost identical to that of the shale-limestone. Moreover, the NMO velocity of the Cotton Valley shale exceeds the vertical rms velocity by 20 percent, whereas the two quantities are almost identical in the shale-limestone. Pending similar study of other models, I conclude from the computations here that transverse isotropy will seldom be an issue of practical importance in DMO processing.

As with other seismic phenomena, stacking velocity behavior in transversely isotropic media differs from that expected for isotropic media in ways that are not readily predictable from one medium to another. Those variations depend on the ratios of the four pertinent elastic moduli-quantities that are neither often nor well determined in rock units, on either a small or large scale. With the derived expression for NMO velocity as a function of reflector dip in a linear $v(z)$, transversely isotropic 
FAI medium, one could do a proper dip correction of stacking velocity, if the elastic moduli and its depth dependence were known. Perhaps, this expression for the dip correction will also give clues as to how DMO could be modified to accommodate both $v(z)$ and anisotropy. In practice, however, our information on the elastic moduli and their variation within the subsurface is meager. Moreover, it is unlikely that the subsurface would rather consistently have the properties of, say, the shale-limestone from surface to dipping reflector. These considerations provide little motivation to develop a DMO process for transversely isotropic media.

\section{ACKNOWLEDGMENTS}

I greatly appreciate several stimulating discussions with Dave Hale, out of which came improved understanding of the implications of stacking-velocity behavior for DMO performance and recognition that stacking velocity for the anisotropic media could be studied efficiently through computation of NMO velocity. Financial support for this work was provided in part by the United States Department of Energy, Grant Number DE-FG02-89ER14079 (this support does not constitute an endorsement by DOE of the views expressed in this paper), and by the members of the Consortium Project on Seismic Inverse Methods for Complex Structures at the Center for Wave Phenomena, Colorado School of Mines.

\section{REFERENCES}

Byun, B., 1984, Seismic parameters for transversely isotropic media: Geophysics, 49, 1908-1914.

Červený V., 1989, Ray tracing in factorized anisotropic inhomogeneous media: Geophys. J. Int., 99, 91-100.

Hubral, P. and Krey, T., 1980, Interval velocities from seismic reflection time measurements: Soc. of Explor. Geophys., Tulsa, OK, 203 p.

Larner, K. and Cohen, 1992, Migration error in transversely isotropic media with linear velocity variation in depth: 62nd Ann. Intl. Mtg. of the Soc. of Explor. Geophys., New Orleans.

Levin, F. K., 1971, Apparent velocity from dipping interface reflections: Geophysics, 36, $510-516$.

Levin, F. K., 1979, Seismic velocities in transversely isotropic media: Geophysics, 44, 918-936.

Levin, F. K., 1990, Reflection from a dipping plane-Transversely isotropic solid: Geophysics, 55, 851-855.

Press, W. H., Flannery, B. P., Teukolsky, S. A., and Vetterling, W. T., 1986, Numerical recipes: Cambridge Univ. Press.

Shah, P. M., 1973, Use of wavefront curvature to relate seismic data with subsurface parameters: Geophysics, 38, 812-825. 
Shearer, P. M. and Chapman, C. H., 1988, Ray tracing in anisotropic media with linear velocity gradient: Geophys. J. Int., 94, 575-580.

Slotnick, M. M., 1986, Lessons in seismic computing: Soc. of Explor. Geophys., $268 \mathrm{p}$.

Thomsen, L., 1986, Weak elastic anisotropy: Geophysics, 51, 1954-1966.

White, J. E., 1983, Underground sound: Application of sound waves: Elsevier, $253 \mathrm{p}$. 

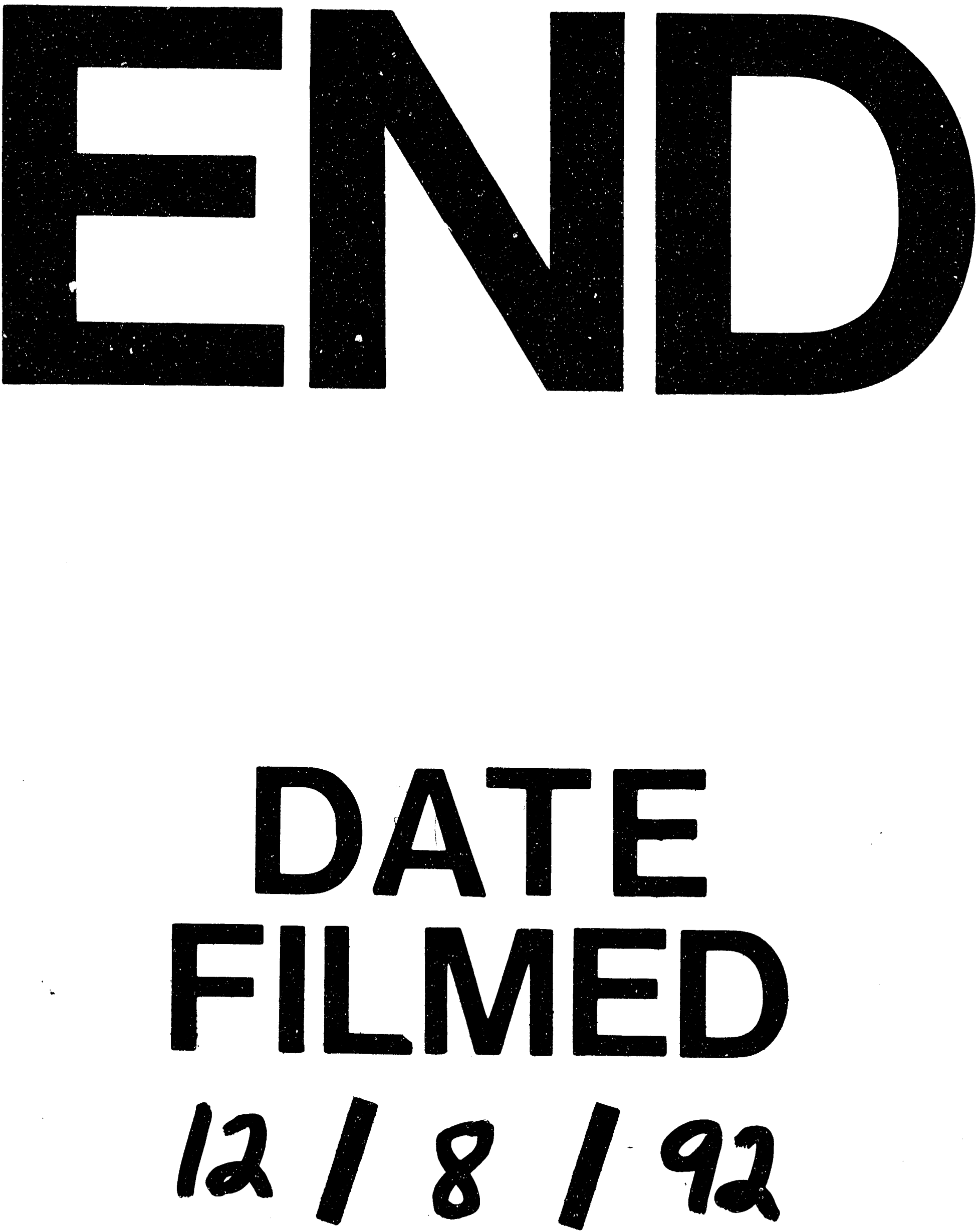
, 